\title{
Jörn Leonhard(dir.),Vergleich und Verflechtung, Deutschland und Frankreich im 20. Jahrhundert
}

\author{
Nicolas Batteux
}

\section{OpenEdition}

Journals

Édition électronique

URL : http://journals.openedition.org/ifha/11224

DOI : 10.4000/ifha. 11224

ISSN : 2198-8943

Éditeur

IFRA - Institut franco-allemand (sciences historiques et sociales)

Référence électronique

Nicolas Batteux, «Jörn Leonhard(dir.),Vergleich und Verflechtung, Deutschland und Frankreich im 20. Jahrhundert », Revue de l'IFHA [En ligne], Date de recension, mis en ligne le 15 avril 2021, consulté le 17 avril 2021. URL : http://journals.openedition.org/ifha/11224 ; DOI : https://doi.org/10.4000/ifha.11224

Ce document a été généré automatiquement le 17 avril 2021

CIFHA 


\title{
Jörn Leonhard(dir.),Vergleich und Verflechtung, Deutschland und Frankreich im 20. Jahrhundert
}

\author{
Nicolas Batteux
}

Le présent ouvrage est le résultat d'un colloque organisé par le Frankreich Zentrum de l'Université de Fribourg-enBrisgau en 2013 à l'occasion du 50e anniversaire du Traité de l'Élysée et publié en 2015, sous la direction de l'historien Jörn Leonhard. Il réunit des contributions de spécialistes reconnus des relations franco-allemandes et de l'histoire de la France et de l'Allemagne venus des deux pays. Une première remarque s'impose sur le titre de l'ouvrage, Vergleich und Verflechtung, qui laisse attendre d'abord une contribution méthodologique théorique, ce que cet ouvrage n'est pas, ou du moins, pas directement. À cet endroit, le sous-titre Deutschland und Frankreich im 20. Jahrhundert renvoie plus précisément à la diversité des thématiques abordées dans

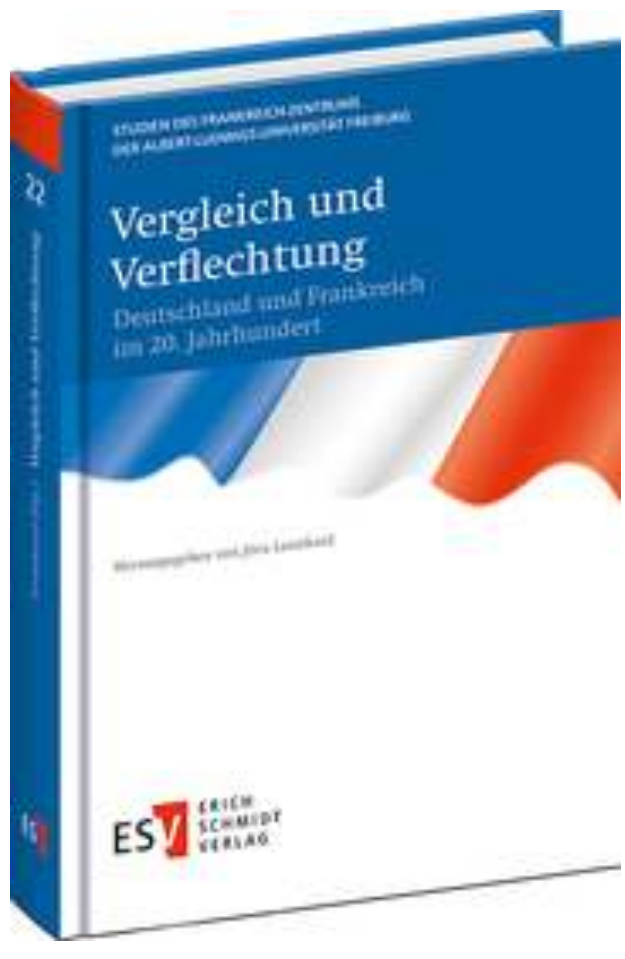
ce volume, qui vont de la politique mémorielle, la culture jeune à la diplomatie et aux systèmes sociaux. La problématique méthodologique intervient donc dans un second temps, dans la confrontation des différents articles, qui adoptent différents outils dans leur traitement de l'objet francoallemand: comparaison, transfert et histoire croisée, comme autant d'approches complémentaires. 
L'ouvrage est divisé en 3 sections, respectivement consacrées à l'arrière-plan historique, au Traité de l'Élysée et à la question du « couple » franco-allemand, et à la période post-1963 sous l'angle de l'histoire diplomatique, culturelle et sociale. À cet endroit, la structuration de l'ouvrage confère au Traité de l'Élysée le caractère de césure, qui n'est néanmoins pas véritablement traité ou questionné dans la dernière partie du volume, 1963 n'étant même (presque) jamais évoqué dans ces contributions.

Dans la première partie de l'ouvrage, consacrée à l'arrière-plan historique, $\mathrm{S}$. Schirmann apporte une vision nuancée des relations franco-allemandes dans l'entredeux-guerres en présentant celles-ci selon une logique non-linéaire, certes faite de tensions, mais aussi de moments de coopération fructueuse. C. Defrance étudie quant à elle les politiques mémorielles dans les deux pays après 1945 et démontre comment le travail de mémoire fut intrinsèquement lié au traitement de cette question par le voisin et comment la politique de réconciliation entre les deux pays a parfois pris le pas sur les enjeux mémoriels. A. Wilkens revient ensuite sur les "options" françaises et allemandes dans la politique européenne dans les années 1950. U. Lappenküpper, dans son analyse de la politique de défense et de sécurité des deux pays, montre également qu'une comparaison franco-allemande ne peut parfois faire l'économie d'acteurs tiers, comme ce fut le cas des États-Unis sur cette question. Pour clôturer cette première partie, R. Hudemann aborde le problème de la décolonisation. Il y montre aussi que la comparaison dispose d'un potentiel heuristique important, même lorsqu'elle est asymétrique, dans la mesure où seule la France fut placée face au défi de la décolonisation après la Seconde Guerre mondiale, l'Empire allemand ayant été liquidé après 1918.

La deuxième partie est quant à elle plus resserrée sur le plan thématique puisqu'elle place la focale sur le Traité de l'Élysée et l'institutionnalisation de l'amitié francoallemande. H. Miard-Delacroix examine tout d'abord cette question sous l'angle de l'histoire des émotions. Elle y analyse le traité à l'aune des catégories du calcul et de l'émotion, non comme des modalités s'excluant mutuellement, mais au contraire, comme des phénomènes concomitants. U. Pfeil décentre ensuite le regard de la seule perspective RFA-France pour étudier la réception est-allemande du traité et les réactions du régime de RDA, marqué par le sentiment du danger permanent. Les contributions de $\mathrm{M}$. Waechter et de $\mathrm{C}$. Hiepel sont quant à elles complémentaires puisqu'elles mettent toutes deux l'accent sur la thématique du «couple francoallemand», M. Waechter s'intéressant aux «binômes" canoniques (De GaulleAdenauer, Giscard d'Estaing-Schmidt et Mitterrand-Kohl), là où C. Hiepel consacre sa contribution au seul «couple» Brandt-Pompidou, le moins célébré et le moins fréquemment mis en avant.

La dernière partie, enfin, examine l'après 1963 dans une perspective davantage axée sur l'histoire sociale et culturelle. C. Wenkel aborde tout d'abord le rôle joué par Mitterrand au moment de l'unité allemande à partir des archives diplomatiques. Les contributions de $\mathrm{H}$. Kaelble et d'U. Kempf comparent classiquement les systèmes sociaux des deux pays, en prenant respectivement en compte les inégalités sociales et l'État social. D. Hüser examine pour sa part l'évolution des cultures jeunes et protestataires en France et en Allemagne entre les années 1950 et 2000. Enfin, T. Raithel s'intéresse au concept de nation dans l'historiographie française et allemande et à son évolution. 
Cet ouvrage s'avère au total extrêmement riche dans ses contenus et dans les méthodes employées. Les différentes contributions témoignent d'un pragmatisme méthodologique lié au choix des objets d'étude historiques et montrent par ailleurs que la comparaison historique garde toute sa pertinence à l'heure de l'histoire globale. Très divers, les articles de ce volume ont très souvent pour point commun la remise en question de grands récits historiques (sur les réticences de Mitterrand face à l'unité allemande, l'engagement européen dans les années 1950...) et montrent l'intérêt de l'histoire transnationale dans la déconstruction de narrations proprement nationales.

INDEX

Thèmes : Histoire des Etats et des pouvoirs, Historiographie/Methodologie

Index chronologique : Epoque contemporaine

\section{AUTEUR}

\section{NICOLAS BATTEUX}

Sorbonne Université - UMR 8138 SIRICE /Rheinische Friedrich-Wilhelms-Universität Bonn 